Research Paper

\title{
Mulberry polyphenol extracts attenuated senescence through inhibition of Ras/ERK via promoting Ras degradation in VSMC
}

\author{
Ching-Pei Chen ${ }^{1 \#, ~ K u e i-C h u a n ~ C h a n ², 3 \#, ~ H s i e h-H s u n ~ H o ~}{ }^{4}$, Hui-Pei Huang5, Li-Sung Hsu ${ }^{6,7 凶}$, Chau-Jong \\ Wang $8,9 \bowtie$ \\ 1. Cardiovascular division of Changhua Cristian Hospital, Changhua, Taiwan. \\ 2. School of Medicine, Chung Shan Medical University, Taichung 402, Taiwan \\ 3. Department of Internal Medicine, Chung Shan Medical University Hospital, Taichung, 402, Taiwan. \\ 4. Institute of Biochemistry and Biotechnology, Chung Shan Medical University, Tai-chung, 402, Taiwan. \\ 5. Department of Biochemistry, School of Medicine, Medical College, Chung Shan Medical University, Taichung, 402, Taiwan. \\ 6. Institute of Medicine, Chung Shan Medical University, Taichung, 402, Taiwan. \\ 7. Clinical Laboratory, Chung Shan Medical University Hospital, Taichung, 402, Taiwan. \\ 8. Department of Health Diet and Industry Management, Chung Shan Medical University, Taichung 402, Taiwan. \\ 9. Department of Medical Research, Chung Shan Medical University Hospital, Taichung, 402, Taiwan. \\ \#: these authors contributed equally as first author. \\ Corresponding authors: Li-Sung Hsu, Tel: 886-4-24730022 ext 11682, E-mail address: 1shsu405@yahoo.com.tw (L. -S. Hsu). Chau-Jong Wang, Tel:
} 886-4-24730022 ext 11670, E-mail address: wcj@csmu.edu.tw (C. -J. Wang)

(C) The author(s). This is an open access article distributed under the terms of the Creative Commons Attribution License (https://creativecommons.org/licenses/by/4.0/). See http://ivyspring.com/terms for full terms and conditions.

Received: 2021.07.09; Accepted: 2021.11.04; Published: 2022.01.01

\begin{abstract}
Ageing is one of the major risk factors of human diseases, including cancer, diabetes, and cardiovascular disease. Mulberry exhibits a wide range of functions, such as anti-oxidant, anti-inflammation, and anti-diabetes. In this study, we investigated the role of mulberry polyphenol extract (MPE) in K-Ras-induced senescence of smooth muscle cells. Forced expression of K-Ras enhanced senescence of smooth muscle A7r5 cells as shown by the elevation of $\beta$-galactosidase activity. Treatment with MPE significantly repressed the Ras, phosphorylated ERK, and $\beta$-galactosidase level. MPE triggered the association of cyclins with their corresponding cyclin-dependent protein kinases and hyperphosphorylated retinoblastoma (RB). MPE also down-regulated the levels of K-Ras-induced CDK inhibitors. MPE enhanced the phosphorylated AMP-dependent protein kinase (AMPK) and inducible nitric oxide synthase (iNOS) level in the presence of K-Ras. Pretreatment with either L-NAME or AMPK inhibitor reversed the effects of MPE. In addition, L-NAME and AMPK inhibitor repressed the MPE-induced total and phosphorylated 3-hydroxy-3-methylglutaryl coenzyme A (HMG-Co A) level. MPE repressed K-Ras-induced G0/GI arrest, whereas L-NAME and AMPK inhibitor blocked the effects of MPE. Our results indicated that MPE recovered the K-Ras-induced senescence of vascular smooth muscle cells through iNOS and AMPK-dependent pathway. Our findings suggested that MPE may prevent ageing-induced atherosclerosis.
\end{abstract}

Key words: mulberry polyphenol extract, Ras, smooth muscle cell, senescence

\section{Introduction}

Ageing is a multiple factor-induced process. Lopez-Otin et al. have categorized nine hallmarks of ageing, namely, genomic instability, telomere attrition, epigenetic alterations, loss of proteostasis, deregulated nutrient sensing, mitochondrial dysfunction, cellular senescence, stem cell exhaustion, and altered intercellular communication [1]. Ageing promotes a wide range of human diseases, such as atherosclerosis, neurodegeneration diseases, and cancers [1]. Scientists focus on developing novel agents to attenuate the ageing process and prevent ageing-related diseases.

Ras proteins play a critical role in several physiological functions, such as cell proliferation, migration, and ageing process [2]. Sun et al.[3] demonstrated that mutation of RAS1 or RAS2 
promoted the life span in Saccharomyces cerevisiae. In fruit fly Drosophila melanogaster, a constitutively activated form of Anterior open (AOP), a transcriptional factor repressed by RAS, contributed to a longer lifespan [4]. Jazwinski et al. demonstrated that genetic variants of HRAS1, apolipoprotein E, and ceramide synthase LASS1 synergistically play a critical role in the longevity and healthy ageing of humans [5].

Recent emerging reports indicated that extracts from traditional herbs slowed the ageing process and treated ageing-related diseases. Exposure to ethanol extracts from the leaves of Humulus japonicus increased the lifespan of yeast cells through the enhanced expression of longevity-related proteins, such as sirtuin 1 and AMP-dependent protein kinase [6]. Administration of ethyl acetate extracts from Physalis alkekengin (PAE) significantly reversed D-galactose-induced ageing-related impairment of neuron functions [7]. Moreover, PAE also attenuated D-galactose triggered senescence by diminished oxidative stress [7]. Treatment with coffee silverskin aqueous extract clearly inhibited tert-butyl hydroperoxide-induced ageing in $\mathrm{HaCaT}$ cells and promoted longer longevity in Caenorhabditis elegan [8]. Mulberry (Morus alba L.) is an economically important plant that is widely distributed in Asia region and extracts from different parts of mulberry enriched in flavonoids possess several biological functions, such as anti-oxidant, anti-obesity, and anti-inflammation [9]. Mulberry leaf extract (MLE) prevented atherosclerosis through the reduction of oxidative low density lipoprotein-elevated reactive oxygen species [10]. Administration of MLE attenuated the obesity-induced non-alcohol fatty liver disease through the regulation of lipogenesis and the elevation of anti-oxidant enzyme expression [11]. A previous report from our laboratory indicated that mulberry water extract induced smooth muscle cells to undergo apoptosis via the activation of both intrinsic and extrinsic pathways and inhibited atherosclerosis in a rabbit model [12]. Moreover, MPE mitigated the migration of $\mathrm{A} 7 \mathrm{r} 5$ cells through the downregulation of FAK/SRC/PI3-K pathway [13]. The molecular mechanisms of MPE on Ras-induced VSMC ageing still remains to be elucidated. In the present study, we investigated the effect of MPE on Ras-induced ageing in A7R5 smooth muscle cells.

\section{Materials and Methods}

\section{Materials}

All chemicals including AMPK inhibitor (Compound C) and L-NAME were purchased from Sigma-Aldrich (St. Louis, MO, USA). Antibodies against p53 (Pab 240), retinoblastoma (RB; G3-245) and E2F (KH95/E2F) were purchased from BD Biosciences (San Jose, CA, USA). Phosphorylate p53 (16G8) and phosphorylated RB (D20B12) antibodies were obtained from Cell Signaling Technology Inc. (Beverly, MA, USA). Anti-phosphorylated ERK (E-4), anti-ERK (C-14), anti-cyclin D1 (HD-11), anti-cyclin E (M-20), anti-cyclin A (H-432), anti- CDK2 (D-12), antiCDK4 (DSC-35), anti- p21 (F-5), anti- p27 (F-8), and anti- p16 (F-12) were purchased from Santa Cruz Biotechnology Inc. (Santa Cruz, CA, USA).

\section{Preparation of MPE and HPLC analysis}

MPE was prepared as previously reported [13]. Dried mulberry fruit powder (100 g) was dissolved in $500 \mathrm{ml}$ methanol and stirred at $50{ }^{\circ} \mathrm{C}$ for $3 \mathrm{~h}$. The debris of methanol extract was removed by filter. The extract was lyophilized under reduced pressure at room temperature. After resuspension in $500 \mathrm{~mL}$ of 50 ${ }^{\circ} \mathrm{C}$ distilled water and extraction with $180 \mathrm{~mL}$ of ethyl acetate thrice, the MPE was redissolved in $300 \mathrm{~mL}$ of distilled water and lyophilized. The MPE powders were stored in $-80^{\circ} \mathrm{C}$ until use. HPLC analysis of MPE was performed as previously reported [13]. The major compounds of MPE included rutin (18.17\%), protocatechuic acid (13.76\%), naringenin (6.71\%), epigallocatechin gallate $(6.26 \%)$, caffeic acid $(6.15 \%)$, quercetin $(5.97 \%)$, and several minor polyphenols, such as epicatechin $(4.69 \%)$, catechin $(3.19 \%)$, gallic acid $(2.67 \%)$, p-coumaric acid $(2.48 \%)$, and hesperetin $(2.08 \%)$ [13].

\section{Cell culture and selection of stable clones}

The rat smooth muscle A7r5 cells obtained from American Type Culture Collection (ATCC, Manassas, VA, USA) were maintained in Dulbecco's modified Eagle's medium (DMEM; HyClone, Marlborough, Massachusetts, USA) supplemented with $10 \%$ fatal bovine serum (Biological Industries, Kibbutz Beit-Haemek, Israel), 1\% glutamine (HyClone, Marlborough, MA, USA), and $1 \%$ penicillinstreptomycin (HyClone, Marlborough, MA, USA). The cells were cultured in an incubator at $37^{\circ} \mathrm{C}$ with a humidified atmosphere of $5 \% \mathrm{CO}_{2}$.

The A7r5 cells were seeded in 6-well plate and transfected with pcDNA3.0 or pcDNA3.0-K-Ras plasmid by Lipofectamine 2000 (ThermoFisher SCIENTIFIC, Inc. Waltham, MA, USA) according to the manufacturer's recommendation. A7r5 cells with a stable expression of K-Ras were selected by G418 (ThermoFisher SCIENTIFIC, Inc. Waltham, MA, USA).

\section{$\beta$-galactosidase activity assay}

The A7r5 cells expressing or not expressing 
K-Ras were seeded in 6-well plate at a density of $3 \times$ $10^{5}$. At days $2,4,8,12$, and 16 , the cells were fixed by a fix solution $(2 \%$ formaldehyde and $0.2 \%$ glutaraldehyde) at room temperature for $5 \mathrm{~min}$. After washing with phosphate buffered saline (PBS), cells were incubated with the staining solution $(40 \mathrm{mM}$ citric acid, sodium phosphate $\mathrm{pH} 6.0,150 \mathrm{mM} \mathrm{NaCl}, 2$ $\mathrm{mM} \mathrm{MgCl} 2$, and $20 \mathrm{mg} / \mathrm{ml} \mathrm{X-gal)}$ at $37^{\circ} \mathrm{C}$ for $12-16 \mathrm{~h}$. Images of the cells were taken using microscopy at $X$ 400 magnification.

\section{Cell cycle analysis}

The A7r5 cells expressing or not expressing K-Ras were treated with MPE in the presence of L-NAME or AMPK inhibitor for $24 \mathrm{~h}$. Cells were detached and fixed by $70 \%$ alcohol overnight at $-20^{\circ} \mathrm{C}$. After washing with cold PBS, cells were incubated with $50 \mu \mathrm{g} / \mathrm{mL}$ propidium iodine (PI) and $100 \mu \mathrm{g} / \mathrm{ml}$ RNase A in PBS for $15 \mathrm{~min}$ in the dark. The cell cycles of stained cells were analyzed by flow cytometer (Becton Dickinson, CA, USA) and CellQuest Software (Becton Dickinson, CA, USA).

\section{Immunoprecipitation assay}

The A7r5 cells were lysed by RIPA buffer containing proteinase inhibitors $(1 \mathrm{mM} / \mathrm{ml} \mathrm{Na} 3 \mathrm{VO} 4$, $1.7 \mu \mathrm{g} / \mathrm{ml}$ leupeptin and $100 \mu \mathrm{g} / \mathrm{ml}$ PMSF). The protein concentration was measured by Bio-Rad protein assay kit. Cell lysate $(500 \mathrm{mg})$ was incubated with indicated antibodies (1:100 dilution) plus $30 \mu \mathrm{l}$ Agarose-G at $4{ }^{\circ} \mathrm{C}$ overnight. The complexes were washed with PBS twice and centrifuged at $8000 \mathrm{rpm}$ for $5 \mathrm{~min}$. The immunocomplexes were subjected to Western blot analysis.

\section{Western blot analysis}

Proteins $(50 \mu \mathrm{g})$ were separated by sodium dodecyl sulfate polyacrylamide gel electrophoresis (SDS-PAGE) and then transferred into polyvinylidene fluoride (PVDF) membrane. The membrane was blocked by phosphate buffered saline (PBS) containing $5 \%$ non-fat milk for $1 \mathrm{~h}$ and incubated with specific antibodies for overnight at $4{ }^{\circ} \mathrm{C}$. The membrane was washed with PBS containing $0.1 \%$ Tween-20 thrice and then incubated with an horseradish peroxidase (HRP)-conjugated second antibody. The positive signals were determined by using an enhanced chemiluminescence kit.

\section{Reverse transcription-polymerase chain reaction ( $R T$ T-PCR)}

The TRIzol reagent was utilized to purify total RNA from A7r5 cells with or without K-Ras transfection in the presence or absence of MPE for indicated time according to the manufacturer's protocols. First strand cDNA was generated by
Moloney Murine Leukemia Virus (M-MLV) reverse transcriptase, oligo-dT primer and $4 \mu \mathrm{g}$ total RA. PCR was performed using K-Ras primers were: Sense: $5^{\prime}$ CTTGATAATCTTGTGTGGAAC-3' and Antisense: 5' -CCTCCCTTTACAAATTGTAC- $3^{\prime}$. GAPDH primers were: sense: 5'-ACCACAGTCCATGCCATCAC-3 and anti-sense: 5'-TCCACCACCCTGTTGCTGTA-3'The PCR condition was: denaturation at $95{ }^{\circ} \mathrm{C}$ for $1 \mathrm{~min}$, annealing at $55^{\circ} \mathrm{C}$ for $1 \mathrm{~min}$, and extension at $72{ }^{\circ} \mathrm{C}$ for 1 min. Total cycle was 30 for K-Ras and 28 for GADPH. The PCR products were separated by agarose gel and pictured.

\section{Statistical analysis}

Data were represented as means \pm standard division obtained from three independent experiments. Data were analyzed by Student $t$ test using SPSS software version 12 (IBM, Armonk, NY, USA). Significant difference was set as $\mathrm{P}<0.05$.

\section{Results}

\section{Overexpression of K-Ras-induced senescence in smooth muscle cells}

Previous report showed the forced expression of K-Ras triggered senescence in smooth muscle cells via the activation of the p53 and ERK pathways [15]. To verify whether K-Ras-induced senescence in smooth muscle cells, we established the smooth muscle A7r5 cell's stable expression of K-Ras. The cell numbers increased before day 8 and significantly decreased after day 12 in the group stably expressing K-Ras compared with the normal control (Fig. 1A). In addition, the ageing marker ( $\beta$-galactosidase) activity was detected at day 8 and clearly increased at days 12 and 16 compared with the vector alone in A7r5 cells (Fig. 1B).

\section{MPE attenuated Ras-induced senescence and signals}

Next, we investigated the effects of MPE on $\mathrm{K}$-Ras-induced ageing in A7r5 cells. Exposure to 0.005 $\mathrm{mg} / \mathrm{mL}$ of MPE obviously decreased $\beta$-galactosidase activity in A7r5 cells stably expressing K-Ras at day 12 (Fig. 2A). The effect of MPE was stronger than MWE. Thus, we selected MPE for further studies.

To address the effects of MPE on the expression of Ras and its signals, we performed Western blot analysis. Ras expression clearly increased at days 8 and 12. In parallel, phosphorylated ERK and $\beta$-galactosidase levels increased in K-Ras-expressing cells. MPE significantly reduced Ras, phosphorylated ERK, and $\beta$-galactosidase expressions (Fig. 2B).

To address whether MPE enhanced the degradation of Ras, immunoprecipitation and 
Western blot analysis were performed. As shown in Fig 2C, significant ubiquitination was found in the presence of MG-132 and MPE co-treatment groups.

Moreover, we also conducted RT-PCR to detect the mRNA level of K-Ras in the presence of MPE. The mRNA level was increased in K-Ras overexpressd A7r5 cells whereas MPE slightly reduced the K-Ras mRNA at day 8 post-transfection (Figure 2D).

\section{MPE reversed K-Ras-induced cell cycle-related proteins' interaction and expression}

Cell cycle arrest is one of the hallmarks of ageing. To determine whether MPE affected the expressions of cell cycle-related proteins, immunoprecipitation and Western blot were conducted. As shown in Fig. 3, the interaction of cyclin-dependent protein kinase 4 (CDK4) with cyclin D1 or D3 was clearly reduced in K-Ras-expressing cells whereas co-treatment with $0.005 \mathrm{mg} / \mathrm{mL}$ MPE enhanced the interaction of CDK4 with cyclin D1 or D3. Similarly, the interaction of CDK2 with cyclin A or E was blocked by K-Ras whereas MPE reversed the interaction. K-Ras diminished phosphorylation of $\mathrm{RB}$ and enhanced the association of E2F with RB. Treatment with MPE enhanced the phosphorylation of $\mathrm{RB}$ and promoted the dissociation of $\mathrm{RB}$ and E2F. Moreover, the increased p53 phosphorylation and enhanced expression of CDK inhibitors, such as p27, p21, and p16, by K-Ras was reversed by MPE (Fig. 3).

\section{MPE affected AMP-dependent protein kinase (AMPK) and inducible nitric oxide synthase (iNOS) expressions}

To detect the effect of MPE on AMPK and iNOS expressions, Western blot analysis was performed. No overt alternation of phosphorylated AMPK and iNOS level was found in K-Ras-expressing A7r5 cells compared with control A7R5 cells. MPE clearly increased the phosphorylated AMPK and iNOS levels in A7r5 cells stably expressing K-Ras (Fig. 4A). Pretreatment with iNOS inhibitor (L-NAME) or AMPK inhibitor reversed the MPE-induced effects (Fig. 4B). Similarly, MPE-induced NO concentration was blocked by the L-NAME and AMPK inhibitor (Fig. 4C).

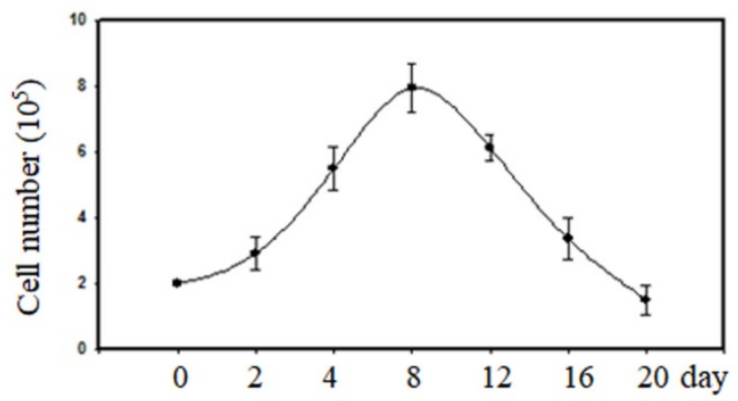

(B)
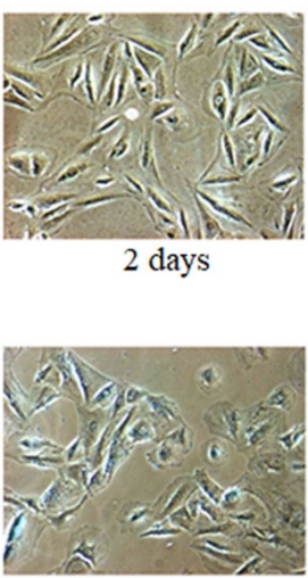

12 days

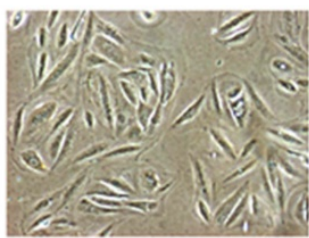

4 days

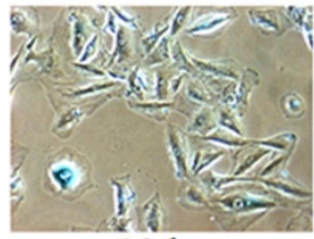

16 days


Vector 8 days

Figure 1. Effect of K-Ras on cell growth and senescence of A7r5 cells. A7r5 cells were with transfected K-Ras and selected by G418 medium for 14 days. (A)The cell were seeded in 6 well plate and maintained for indicated time course. Cell numbers were measured by cell counter. (B) The senescence was detected by $\beta$-galactosidase assay. 
(A)

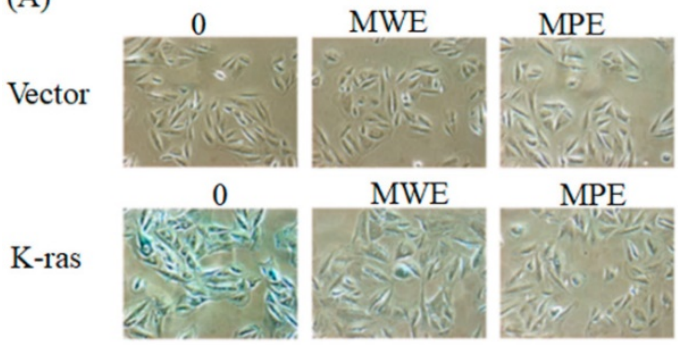

(B)

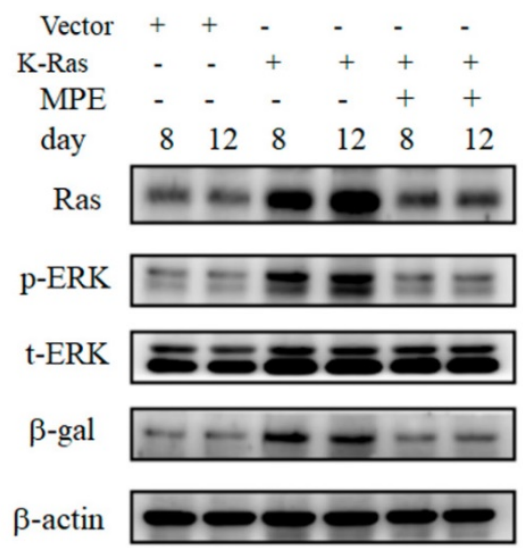

(C)

$\begin{array}{rlllll}\text { Vector }+ & + & - & - & - & - \\ \text { K-Ras }- & - & + & + & + & + \\ \text { MPE }- & - & - & - & + & + \\ \text { MG } 132+ & + & + & + & + & + \\ \text { Day } 8 & 12 & 8 & 12 & 8 & 12\end{array}$

IB: ub

IP: Ras

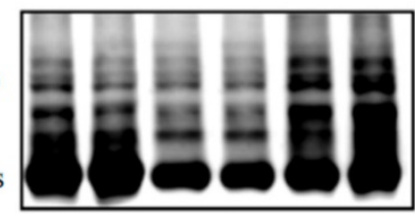

actin



(D)

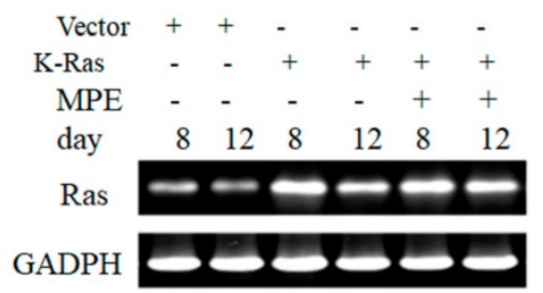

Figure 2. MPE blocked K-Ras-induced senescence and downstream Ras signals of A7r5 cells. (A) A7r5 cells were transfected with K-Ras and treated with MWE or MPE at indicated concentration for 12 days and senescence level was detected by $\beta$-galactosidase assay. (B) Western blot analysis was conducted to detect the expresso of indicated proteins expression of $A 7 \mathrm{r} 5$ cells and stable expressed K-Ras A7r5 cell with or without MPE treatment at day 8 and 12. (C) A7r5 cells and stable expressed K-Ras A7r5 cell with or without MPE treatment at day 8 and 12 in the presence or absence of MG-132 were subjected to perform immunoprecipitation by anti-Ras antibody and Western blot with anti-Ras antibody. Actin was used as internal control. (D) Total RNAs derived from A7r5 cells and stable expressed K-Ras A7r5 cell with or without MPE treatment at day 8 and 12 were subjected into RT-PCR analysis of K-Ras. GAPDH was used as internal control.
(A)

$\begin{array}{ccccccc}\text { Vector } & + & + & - & - & - & - \\ \text { K-Ras } & - & - & + & + & + & + \\ \text { MPE } & - & - & - & - & + & + \\ \text { day } & 8 & 12 & 8 & 12 & 8 & 12\end{array}$

IB

Cyclin 1

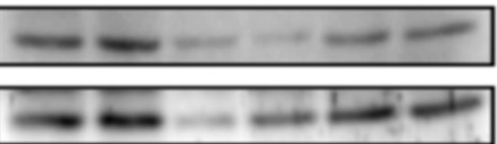

Cyclin D3

IP $\quad$ CDK 4

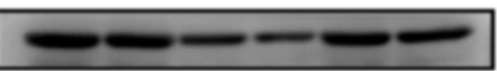

IB
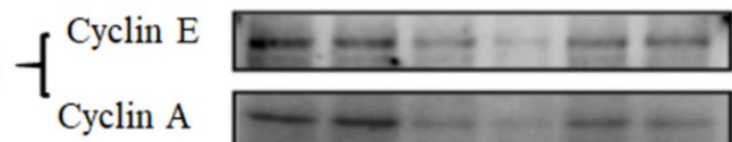

IP $\mathrm{CDK} 2$

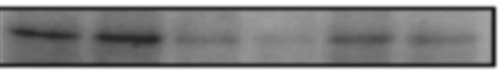

(B)

$\begin{array}{lllllll}\text { Vector } & + & + & - & - & - & - \\ \text { K-Ras } & - & - & + & + & + & + \\ \text { MPE } & - & - & - & - & + & + \\ \text { day } & 8 & 12 & 8 & 12 & 8 & 12\end{array}$

IB


E2F

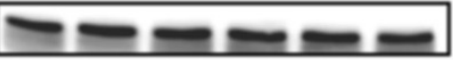

(C)

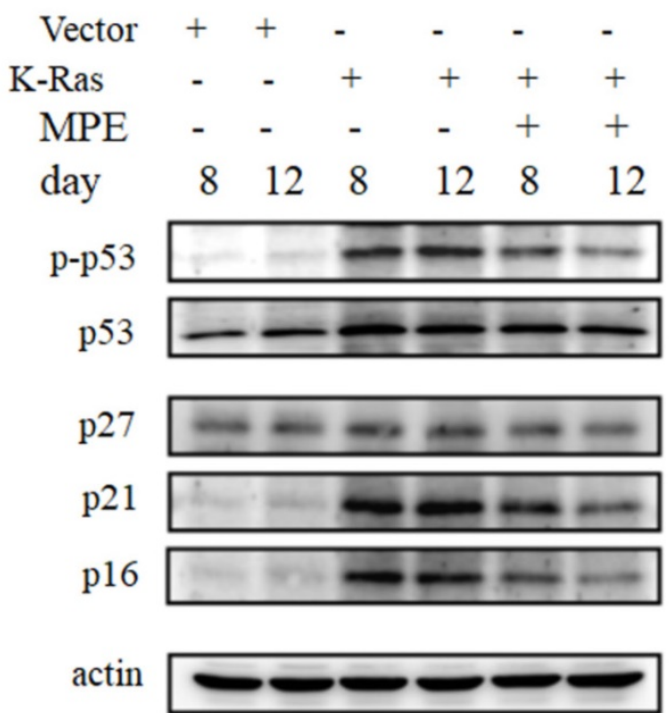

Figure 3. MPE affected cell cycle-related proteins expression. (A) Protein extracts derived from A7r5 cells and stable expressed K-Ras A7r5 cell with or without MPE treatment at day 8 and 12 were subjected into immunoprecipitation with anti-CDK4 (upper panel) or anti-CDK2 (low panel) and then performed Western blot analysis using indicated antibodies. (B) Western blot analysis of indicated CDFK inhibitors of protein extracts derived from A7r 5 cells and stable expressed K-Ras A7r5 cell with or without MPE treatment at day 8 and 12. 
(A)



(B)



(C)





Figure 4. MPE enhanced the activation of AMPK and iNOS. (A) Protein extracts derived from A7r5 cells and stable expressed K-Ras A7r5 cell with or without MPE treatment at day 8 and 12 were subjected into Western blot analysis using antibodies against iNOS, phosphorylated AMPK (p-AMPK), and AMKP. (B) Protein extracts derived from A7r5 cells and stable expressed K-Ras A7r5 cell with or without MPE in the presence of L-NAME or AMPK inhibitor were subjected into Western blot analysis using antibodies against iNOS, phosphorylated AMPK (p-AMPK), and AMKP. Anti-actin was used as internal control. (C) The nitric oxide concentration of A7r5 cells and stable expressed K-Ras A7r5 cell with or without MPE in the presence of L-NAME or AMPK inhibitor were detected. Data represented means standard division from at least three independent experiments. 8 denoted $\mathrm{P}<$ 0.05 ; ** denoted $\mathrm{P}<0.001$.

\section{AMPK and iNOS inhibitor attenuated the effects of MPE}

To verify the effects of AMPK and NOS inhibitor on K-Ras-induced ageing in the presence of MPE, $\beta$-galactosidase activity was determined, and Western blot analysis was performed. Increased $\beta$ galactosidase activity was found in K-Ras-expressing A7r5 cells treated with AMPK and NOS inhibitor in the presence of MPE (Fig. 5A). MPE's blockage of K-Ras-induced phosphorylated p53, total p53, p27, p21, and p16 expressions were reversed by AMPK inhibitor and L-NAME (Fig. 5B). Moreover, MPE also recovered the K-Ras, phosphorylated ERK, and $\beta$-galactosidase expressions (Fig. 5C). In addition, MPE elevated the expression and phosphorylated status of HMG-CoA reductase whereas AMPK and iNOS inhibitors reversed the phenomenon (Fig. 5D).

Cell cycle analysis was used to detect the effects of AMPK and NOS inhibitor on MLE repressed cell cycle arrest. As shown in Fig. 5E, the stable expression of K-Ras in A7r5 cells induced 1.4-fold of G0/G1 arrest compared with the control. Exposure to MPE attenuated the arrest to 1.1-fold, whereas co-treatment with AMPK or NOS inhibitor recovered the cell cycle arrest to 1.3-fold in the presence of K-Ras.

\section{Discussion}

Ageing is one of the major risk factors for atherosclerosis, dementia, and cancer. Prevention of the ageing process is an emerging health issue. Supplementing the diet with flavonoids has been shown to attenuate the ageing process. In this study, we demonstrated that MPE diminished K-Rasinduced ageing in vascular smooth muscle A7r5 cells. MPE significantly attenuated the expression and downstream targets of $\mathrm{K}-\mathrm{Ras}$ in $\mathrm{A} 7 \mathrm{r} 5$ cells. MPE promoted the progression of cell cycle through the enhancement of CDK activities and the downregulation of CDK inhibitors, such as p53, p21, and p16. Moreover, AMPK and iNOS were involved in MPE-mediated functions.

Ras family proteins play pivotal roles in diverse cellular functions, including cell proliferation, cell differentiation, senescence, and metastasis [2]. Knockdown of RAS1 and RAS2 prolonged the replication and chronological lifespan in yeast, respectively [3]. In human smooth muscle cells, activated Ras ((H-rasV12) significantly induced senescence, as evidenced by the elevated senescence-associated $\beta$-galactosidase activity [14]. Futami et al. [15] demonstrated that the forced expression of wild-type or constitutively activated hras in zebrafish Epithelioma papulosum cyprinid (EPC) cells caused senescence by increasing the p53 
expression and $\beta$-galactosidase activity. In line with these observations, our results demonstrated that K-Ras obviously enhanced $\beta$-galactosidase activity and triggered senescence in A7r5 cells.

Using traditional Chinese medicine as anti-ageing molecules has received attention. Mulberry leaf polyphenol affected the transcriptional factors, such as DAF-12, DAF-16, PHA-4, and NHR-80; it controlled the downstream targets expression and eventually prevented ageing in C. elegans [16]. Administration of black mulberry extracts diminished oxidative stress and alleviated cognitive impairment in D-galactose-induced ageing mice [17]. The major components in mulberry extracts also delayed the ageing process. Administration of rutin significantly delayed diabetes-induced ageing in rat CS collagen [18]. Rutin suppressed lipid peroxidation and reduced inflammation in D-glucose-induced ageing mice [19]. In old fibroblast cells, quercetin significantly increased the expression of anti-ageing genes, including growth differentiation factor 11 (GDF11) and Sirt1 [20]. In the present study, we showed that MPE prevented the ageing process through downregulation of K-Ras and its downstream targets.

Senescence, an irreversible cell cycle arrest phenomenon, is one of the hallmarks of ageing [1]. Cell cycle progression is tightly controlled by cyclin-dependent protein kinases (CDKs) and their negative regulators [21]. We demonstrated that MPE increased the association between CDK4/cyclin Ds and CDK2/cyclin E or cyclin A. Moreover, MPE also repressed K-Ras-enhanced CDK inhibitor expression. Quercetin significantly attenuated the expression of p53 in the pancreatic tissues of D-galactose-induced ageing rat [22]. Administration of chlorogenic acid dose-dependently suppressed the vascular senescence of C57/BL6 female mice through the downregulation of p53 and p21 expressions [23]. Our results indicated that MPE promoted cell cycle progression by controlling the association of CDKs/cyclins and the expression of CDK inhibitors.
(A)

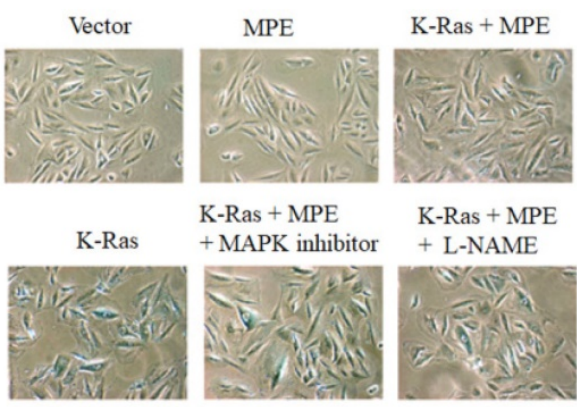

(B)

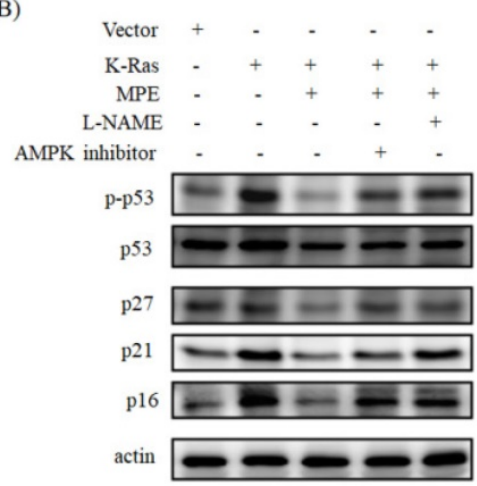

(C)

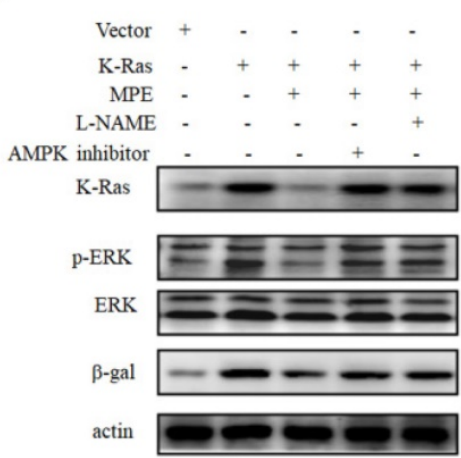

(D)

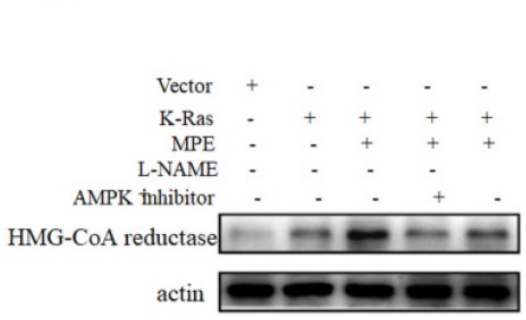

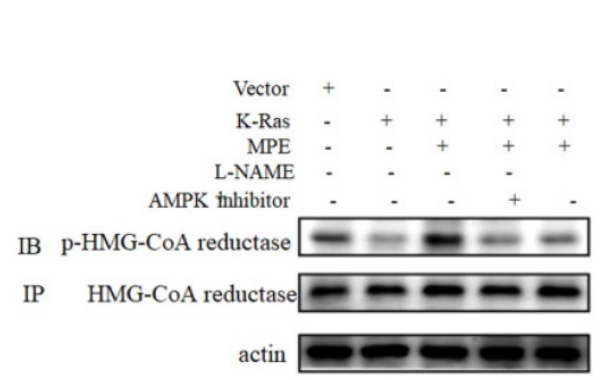

AMPK inhibito
(E)

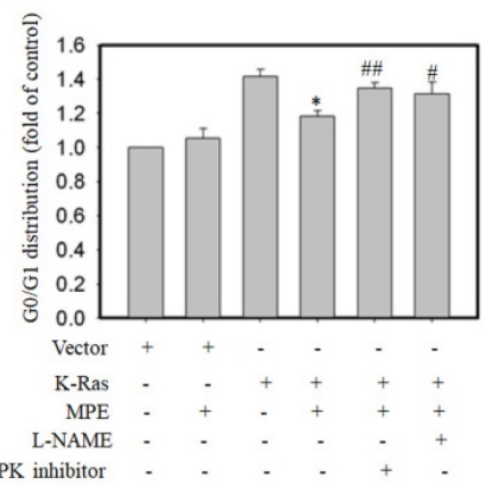

Figure 5. iNOS inhibitor (L-NAME) or AMPK inhibitor blocked anti-senescence of MPE in K-Ras overexpressed A7r5 cells. (A) $\beta$-galactosidase activity was performed in K-Ras stable expressed A7r5 cells or parental cells with or without MPE treatment and indicated inhibitors. (B and C) Protein extracts derived from A7r5 cells and stable expressed K-Ras A7r5 cell with or without MPE in the presence of L-NAME or AMPK inhibitor were subjected into Western blot analysis using indicated antibodies. Anti-actin was used as internal control. (D) Expression and phosphorylated HMG-Co A reductase was analyzed by Western blot and immunoprecipitation, respectively. (E) The G0/G1 distribution of A7r5 cells and stable expressed K-Ras A7r5 cell with or without MPE in the presence of L-NAME or AMPK inhibitor was detected by flow cytometry analysis. Data represented means standard division from at least independent experiments. * denoted P $<0.05$ compared to stable expressed K-Ras A7r 5 cell. \# and \# denoted $\mathrm{P}<0.001$ and 0.05 compared to stable expressed K-Ras A7r5 cell with MPE treatment. 
AMPK is a key sensor for the determination of cellular energy homeostasis [24]. Through regulation of metabolism, AMPK is involved in a wide range of cellular functions, such as apoptosis, autophagy, and longevity [24]. Activation of AMPK homologs enhances the transcription activities of Forkhead box protein $\mathrm{O}$ (FOXO) and peroxisome proliferatoractivated receptor gamma coactivator 1-alpha (PGC-1 $\alpha$ ) and extended the life span of C. elegans [25] Activation of AMPK by quercetin ameliorated $\mathrm{H} 2 \mathrm{O} 2$-induced cell senescence in vascular smooth muscle cells, as evidenced by decreased $\beta$-galactosidase activity and repression of the expressions of CDK inhibitors, such as p53, p21, and p16 [26]. Kou et al. demonstrated that ampelopsin elevated the activity of AMPK/PGC- $1 \alpha$ signaling to prevent skeletal muscle atrophy in D-galactose-induced ageing rat [27]. In the present study, our results showed that MPE obviously stimulated the activity of AMPK and then diminished the K-Ras-induced senescence of A7r5 cells, whereas the phenomenon was blocked by AMPK inhibitor. Our findings indicated that AMPK played a critical role in MPE-induced anti-ageing processes.

Endothelial dysfunction, which means reduction of the synthesis of endothelial NO, is accompanied by ageing and leads to the development of cardiovascular diseases [28]. In the elderly, the inhibition of arginase leads to the elevation of NO-ameliorated endothelial function in an age-dependent manner [29]. Ageing-induced NO level was recovered by treatment with (-)-epicatechin $(1 \mu \mathrm{M})$ for $48 \mathrm{~h}$ in primary bovine coronary artery endothelial cells [30]. Administration of (-)-epicatechin (1 mg kg-1 day-1) for 15 days also increased the NO in rats compared with the group treated with water alone [30]. Herein, we demonstrated that MPE significantly enhanced the activity of iNOS and increased NO level in A7r5 cells stably expressing K-Ras.

\section{Conclusion}

In summary, MPE triggered the activity of iNOS synthase and elevated NO level. NO promoted AMPK activation and attenuated HMG-CoA reductase activity by phosphorylation. MPE blocked Ras-induced senescence of vascular smooth muscle A7r5 cells through the downregulaton of CDK inhibitors and ERK activation. In conclusion, our findings suggested that MPE could be a potential anti-atherosclerosis agent that acts by inhibiting Ras-induced senescence and ageing.

\section{Acknowledgements}

This study was supported by Chung Shan
Medical University and Chuanghua Cristian Hospital with the grant number of CSMU-CCH-109-01 and Chung Shan Medical University Hospital with the grant number of CSH-2018-C-012.

\section{Author Contributions}

Ching-Pei Chen, Kuei-Chuan Chan, and Hsieh-Hsun Ho designed the study. Hsieh-Hsun Ho and Hui-Pei Huang performed the experiments. Li-Sung $\mathrm{Hsu}$ and Chau-Jong Wang wrote and discussed the manuscript. All authors read and approved the final manuscript.

\section{Competing Interests}

The authors have declared that no competing interest exists.

\section{References}

1. Lopez-Otin C, Blasco MA, Partridge L, Serrano M, Kroemer G. The hallmarks of aging. Cell 2013; 153: 1194-217.

2. Slack C. Ras signaling in aging and metabolic regulation. Nutr Healthy Aging 2017; 4: 195-205.

3. Sun J, Kale SP, Childress AM, Pinswasdi C, Jazwinski SM. Divergent roles of RAS1 and RAS2 in yeast longevity. J Biol Chem 1994; 269: 18638-45.

4. Alic N, Giannakou ME, Papatheodorou I, Hoddinott MP, Andrews TD, Bolukbasi E, Partridge L. Interplay of dFOXO and two ETS-family transcription factors determines lifespan in Drosophila melanogaster. PLoS Genet 2014; 10: e1004619.

5. Jazwinski SM, Kim S, Dai J, Li L, Bi X, Jiang JC, Arnold J, Batzer MA, Walker JA, Welsh DA, Lefante CM, Volaufova J, Myers L, Su LJ, Hausman DB, Miceli MV, Ravussin E, Poon LW, Cherry KE, Welsch MA, Georgia Centenarian S, the Louisiana Healthy Aging S. HRAS1 and LASS1 with APOE are associated with human longevity and healthy aging. Aging Cell 2010; 9: 698-708.

6. Sung B, Chung JW, Bae HR, Choi JS, Kim CM, Kim ND. Humulus japonicus extract exhibits antioxidative and anti-aging effects via modulation of the AMPK-SIRT1 pathway. Exp Ther Med 2015; 9: 1819-1826.

7. Sun K, Sun Y, Li H, Han D, Bai Y, Zhao R, Guo Z. Anti-Ageing Effect of Physalis alkekengi Ethyl Acetate Layer on a d-galactose-Induced Mouse Model through the Reduction of Cellular Senescence and Oxidative Stress. Int J Mol Sci 2020; 21 :

8. Iriondo-DeHond A, Martorell P, Genoves S, Ramon D, Stamatakis K, Fresno M, Molina A, Del Castillo MD. Coffee Silverskin Extract Protects against Accelerated Aging Caused by Oxidative Agents. Molecules 2016; 21:

9. Chan EW, Lye PY, Wong SK. Phytochemistry, pharmacology, and clinical trials of Morus alba. Chin J Nat Med 2016; 14: 17-30.

10. Yang MY, Huang CN, Chan KC, Yang YS, Peng CH, Wang CJ. Mulberry leaf polyphenols possess antiatherogenesis effect via inhibiting LDL oxidation and foam cell formation. J Agric Food Chem 2011; 59: 1985-95.

11. Peng CH, Lin HT, Chung DJ, Huang CN, Wang CJ. Mulberry Leaf Extracts prevent obesity-induced NAFLD with regulating adipocytokines, inflammation and oxidative stress. J Food Drug Anal 2018; 26: 778-787.

12. Chan $\mathrm{KC}$, Ho $\mathrm{HH}$, Lin $\mathrm{MC}$, Wu CH, Huang $\mathrm{CN}$, Chang WC, Wang CJ. Mulberry water extracts inhibit rabbit atherosclerosis through stimulation of vascular smooth muscle cell apoptosis via activating p53 and regulating both intrinsic and extrinsic pathways. J Agric Food Chem 2014; 62: 5092-101.

13. Yu MH, Yang TY, Ho HH, Huang HP, Chan KC, Wang CJ. Mulberry Polyphenol Extract Inhibits FAK/Src/PI3K Complex and Related Signaling To Regulate the Migration in A7r5 Cells. J Agric Food Chem 2018; 66: 3860-3869.

14. Minamino $\mathrm{T}$, Yoshida $\mathrm{T}$, Tateno $\mathrm{K}$, Miyauchi $\mathrm{H}$, Zou $\mathrm{Y}$, Toko $\mathrm{H}$, Komuro I. Ras induces vascular smooth muscle cell senescence and inflammation in human atherosclerosis. Circulation 2003; 108: 2264-9.

15. Futami K, Aoyama K, Fukuda K, Maita M, Katagiri T. Increased expression of hras induces early, but not full, senescence in the immortal fish cell line, EPC. Gene 2021; 765: 145116

16. Zheng S, Liao S, Zou Y, Qu Z, Shen W, Shi Y. Mulberry leaf polyphenols delay aging and regulate fat metabolism via the germline signaling pathway in Caenorhabditis elegans. Age (Dordr) 2014; 36: 9719.

17. Turgut NH, Mert DG, Kara H, Egilmez HR, Arslanbas E, Tepe B, Gungor H, Yilmaz N, Tuncel NB. Effect of black mulberry (Morus nigra) extract treatment on cognitive impairment and oxidative stress status of D-galactose-induced aging mice. Pharm Biol 2016; 54: 1052-64.

18. Odetti PR, Borgoglio A, De Pascale A, Rolandi R, Adezati L. Prevention of diabetes-increased aging effect on rat collagen-linked fluorescence by aminoguanidine and rutin. Diabetes 1990; 39: 796-801. 
19. Yang YC, Lin HY, Su KY, Chen CH, Yu YL, Lin CC, Yu SL, Yan HY, Su KJ, Chen YL. Rutin, a Flavonoid That Is a Main Component of Saussurea involucrata, Attenuates the Senescence Effect in D-Galactose Aging Mouse Model. Evid Based Complement Alternat Med 2012; 2012: 980276.

20. Okada $Y$, Okada M. Quercetin, caffeic acid and resveratrol regulate circadian clock genes and aging-related genes in young and old human lung fibroblast cells. Mol Biol Rep 2020; 47: 1021-1032.

21. Coller HA. Regulation of Cell Cycle Entry and Exit: A Single Cell Perspective. Compr Physiol 2019; 10: 317-344.

22. El-Far AH, Lebda MA, Noreldin AE, Atta MS, Elewa YHA, Elfeky M, Mousa SA. Quercetin Attenuates Pancreatic and Renal D-Galactose-Induced Aging-Related Oxidative Alterations in Rats. Int J Mol Sci 2020; 21:

23. Hada Y, Uchida HA, Otaka N, Onishi Y, Okamoto S, Nishiwaki M, Takemoto R, Takeuchi H, Wada J. The Protective Effect of Chlorogenic Acid on Vascular Senescence via the Nrf2/HO-1 Pathway. Int J Mol Sci 2020; 21:

24. Burkewitz K, Zhang $Y$, Mair WB. AMPK at the nexus of energetics and aging. Cell Metab 2014; 20: 10-25.

25. Zhang S, Li F, Zhou T, Wang G, Li Z. Caenorhabditis elegans as a Useful Model for Studying. Aging Mutations. Front Endocrinol (Lausanne) 2020; 11: 554994

26. Kim SG, Sung JY, Kim JR, Choi HC. Quercetin-induced apoptosis ameliorates vascular smooth muscle cell senescence through AMP-activated protein kinase signaling pathway. Korean J Physiol Pharmacol 2020; 24: 69-79.

27. Kou X, Li J, Liu X, Yang X, Fan J, Chen N. Ampelopsin attenuates the atrophy of skeletal muscle from d-gal-induced aging rats through activating AMPK/SIRT1/PGC-1alpha signaling cascade. Biomed Pharmacother 2017; 90: 311-320.

28. Sun HJ, Wu ZY, Nie XW, Bian JS. Role of Endothelial Dysfunction in Cardiovascular Diseases: The Link Between Inflammation and Hydrogen Sulfide. Front Pharmacol 2019; 10: 1568.

29. Mahdi A, Pernow J, Kovamees O. Arginase Inhibition Improves Endothelial Function in an Age-Dependent Manner in Healthy Elderly Humans. Rejuvenation Res 2019; 22: 385-389.

30. Ramirez-Sanchez I, Mansour C, Navarrete-Yanez V, Ayala-Hernandez M, Guevara G, Castillo C, Loredo M, Bustamante M, Ceballos G, Villarreal FJ. (-)-Epicatechin induced reversal of endothelial cell aging and improved vascular function: underlying mechanisms. Food Funct 2018; 9: 4802-4813. 